\title{
La laïcité française, une exception historique, des principes partagés
}

Valentine Zuber

\section{OpenEdition}

1 Journals

Édition électronique

URL : http://journals.openedition.org/rdr/305

DOI : $10.4000 /$ rdr.305

ISSN : 2534-7462

Éditeur

Presses universitaires de Strasbourg

\section{Édition imprimée}

Date de publication : 1 mai 2019

Pagination : 193-205

ISBN : 979-10-344-0045-4

ISSN : 2493-8637

Référence électronique

Valentine Zuber, «La laïcité française, une exception historique, des principes partagés », Revue du droit des religions [En ligne], 7 | 2019, mis en ligne le 09 octobre 2019, consulté le 10 décembre 2020. URL : http://journals.openedition.org/rdr/305 ; DOI : https://doi.org/10.4000/rdr.305

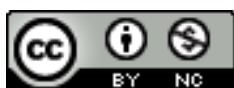

La revue du droit des religions est mise à disposition selon les termes de la Creative Commons Attribution - Pas d'Utilisation Commerciale 4.0 International - CC BY-NC 4.0. 


\section{LA LAÏCITÉFRANÇAISE, UNEEXCEPTION HISTORIQUE, DESPRINCIPESPARTAGÉS}

\section{Valentine ZUBER}

EPHE-PSL/CNRS, Groupe Sociétés, Religions, Läiciés (GSRL)

\section{RÉSUMÉ}

Il est devenu d'usage courant, tant en France qu'à l'étranger, de parler de la laïcité comme d'une exception française qui ne pourrait être transposée dans d'autres cultures que la nôtre. Cette mise au point historique veut au contraire démontrer que si le processus de laicisation français a bien suivi une histoire particulière (dont les grandes étapes sont ici brièvement rappelées), il ne peut s'envisager cependant qu'en lien avec le grand mouvement de sécularisation ayant affecté tous les États-nations modernes, au lendemain des Traités de Westphalie (1648). La laicité, que le terme soit effectivement utilisé ou non, est devenue un principe partagé par tous les États de droit qui reconnaissent, à travers un fonctionnement démocratique de leurs institutions, le pluralisme à l'œuvre dans leur société.

\section{ABSTRACt}

It has become common practice, both in France and abroad, to speak of laïcité as a French exception that could not be transposed into other cultures. On the contrary, this historical review aims to demonstrate that while the process of French secularization has indeed followed a particular history (the main stages of which are briefly recalled here), it can only be considered in relation to the great secularization movement that affected all modern nation-states after the Treaties of Westphalia (1648). Laïcité, whether or not the term is actually used, has become a principle shared by all States ruled by law which recognize, through the democratic functioning of their institutions, the pluralism at work in their society. 
L a laïcité s'appuie conceptuellement sur l'idée de la séparation des sphères temporelles et spirituelles contenue dans le message évangélique qui intime aux hommes de « rendre à César ce qui est à César et à Dieu ce qui est à Dieu» (Matthieu, XXII,21). Mais c'est surtout un concept politique - essentiellement issu de la modernité occidentale - qui est apparu au lendemain de la partition de la chrétienté médiévale. Il a lentement émergé au sein des sociétés européennes à partir $\mathrm{du} \mathrm{XVI}^{\mathrm{e}}$ siècle marqué par de longues et sanglantes guerres politico-religieuses. Ses modalités d'application se sont progressivement forgées dans les réflexions juridico-politiques émises par toute une série d'auteurs qui ont successivement tenté de penser l'autonomie de l'État moderne et la manière dont celui-ci pouvait régir pacifiquement la pluralité religieuse apparue au lendemain de la partition confessionnelle de la chrétienté occasionnée par l'irruption traumatique de la Réforme protestante.

L'idée (sinon le terme) de laïcité s'est affirmée en même temps que se sont construit les États-nations au lendemain des Traités de Westphalie de 1648. Ces derniers qui soldaient la guerre de Trente Ans, qui avait bouleversé la géopolitique européenne, ont contribué à définir une toute nouvelle conception de la souveraineté nationale. Les traités reconnaissaient par ailleurs l'existence légitime et légale de trois confessions chrétiennes différentes en Europe, la catholique, la luthérienne et la calviniste. Ils réservaient aux princes le droit d'imposer leur propre choix religieux à tous leurs sujets et faisaient du domaine religieux une prérogative de l'État souverain qui avait le droit de le gérer librement sans craindre de contrainte internationale. Cette laïcisation progressive des relations internationales a permis dans un premier temps, et au grand mécontentement du Saint-Siège (catholique), de s'émanciper des dogmes religieux dans leurs relations diplomatiques.

Dans les pays protestants, les princes sont généralement devenus les chefs d'Églises nationales indépendantes en subordonnant à leur pouvoir politique toutes les prérogatives religieuses. Dans les pays catholiques, les souverains, toujours théoriquement soumis à la souveraineté spirituelle du pape, chef de l'Église universelle, se sont peu à peu arrogé la direction et les modalités d'organisation du culte catholique sur leur territoire, selon des principes régaliens. Tous les princes d'Ancien Régime ont de plus procédé à une première laïcisation du droit qui a permis de progressivement détacher un ensemble de règles politiques et civiles des prescriptions de nature proprement religieuse.

Ce sont les principes politiques issus des Révolutions américaine et française qui ont permis d'approfondir encore la laïcisation irréversible des États-nations modernes. Substituant à la traditionnelle tolérance civile des 
minorités religieuses le principe de liberté religieuse accordée à tous les sujets ou citoyens d'un État, ces principes ont permis la reconnaissance institutionnelle d'une véritable citoyenneté - égale pour tous - et indépendante de l'identité religieuse propre à chacun. Ce divorce inéluctable entre l'État moderne et les religions s'est accompli de manière soit relativement brutale, soit plus progressive. Dans le premier cas on parle de processus de laïcisation, car c'est l'État qui s'est auto-émancipé dans le cadre d'une lutte politique déclarée face aux prétentions de l'Église catholique en ce domaine. La laiicité y a été instaurée autoritairement par des lois civiles (création d'un état-civil, de systèmes - juridique, de santé et d'éducation - étatiques qui ont permis la dissociation nette entre lois civiles et lois religieuses). Dans

le deuxième cas, on parle plutôt de processus de sécularisation car les religions traditionnellement soumises d'un point de vue légal à l'État se sont progressivement sécularisées en même temps que ce dernier qui n'a pas eu besoin de l'imposer (acceptation du pluralisme doctrinal et du fonctionnement démocratique en leur sein).

Le contenu de la sphère proprement laïque garantie par l'État s'est précisé et enrichi au fil des années, à des rythmes différents selon les espaces considérés. Cela s'est illustré par la fin des discriminations raciales ou religieuses, l'abolition de l'esclavage, la naissance d'un état-civil laïque, la mise en place d'un mariage et d'un divorce civil, l'égalité civile et politique reconnue à tous les citoyens (jusqu'au droit de vote accordé aux femmes), la mise en place d'une éducation régie par l'État, d'une justice unique applicable à tous et l'organisation étatique de la santé. Ce transfert progressif des compétences sociales autrefois dévolues aux différentes Églises a affecté l'ensemble des États modernes au cours des $\mathrm{XIX}^{e}$ et $\mathrm{Xx}^{e}$ siècles. Il a accompagné le développement économique et social propice à l'apparition des sociétés civiles et a contribué à incarner la mise en place de l'idéal démocratique et libéral. Le principe de laïcité est désormais une réalité partagée dans plusieurs pays dans le monde, essentiellement cependant dans les États de droit qui se reconnaissent dans un fonctionnement démocratique et qui acceptent l'expression sans restriction du pluralisme religieux et convictionnel dans la société.

\section{PETITE HISTOIRE DE LA LAÏCITÉ FRANÇAISE}

Le néologisme «laïcité» apparaît assez tard en France au cours du $\mathrm{XIX}^{\mathrm{e}}$ siècle, au moment des débats sur la laïcisation de l'État, c'est-à-dire de son émancipation politique vis-à-vis des principes religieux de l'Église catholique. 
Ferdinand Buisson, l'un des principaux collaborateurs de Jules Ferry, donne en 1882 la première définition de l'État laïque dans son article «Laïcité » tiré du Dictionnaire de pédagogie: "L'État laïque est un État neutre entre les cultes, indépendant de tous les clergés, dégagé de toute conception théologique.»

Le processus de laïcisation à la française s'est appuyé sur trois principes politiques définis progressivement au cours de l'histoire de cette nation.

Le premier principe énonce l'autonomie du politique. Le pouvoir n'est pas l'émanation de la volonté divine, mais le représentant d'une communauté de citoyens apte à se gouverner par elle-même. Le deuxième principe postule la privatisation inéluctable du religieux. Le dispositif républicain ne met pas en cause la liberté de conscience, mais les Églises ne doivent relever exclusivement que du droit privé. Le troisième principe affirme la suréminence de l'État par rapport à la société civile. Le citoyen doit participer à l'élaboration d'une identité collective, qui ne peut se construire que dans l'arrachement à l'appartenance immédiate, sous les auspices de la raison seule.

Ce modèle de laïcité théorisé par les philosophes des Lumières et mis en application depuis la Révolution française se heurte actuellement aux défis du monde ultramoderne. Avec la mondialisation des produits et des idées, le relativisme croissant des valeurs - caractéristique de la société occidentale depuis les années 1960 - n'épargne pas la société française. Les valeurs prônées par le modèle de la République traditionnelle sont de plus en plus contestées par une part croissante de la population. Les principaux piliers de l'éthique laïque perdent de leur pertinence (i.e. le Progrès, la Nation, la Raison). De nouveaux combats surgissent qui mobilisent le concept de laïcité de manière inédite (comme le combat pour la sanctuarisation de l'école ou pour la défense du droit des femmes). L'exaltation des différences et la montée en puissance de l'individualisme rendent aussi de plus en plus difficile la soumission de tous à une norme abstraite et unifiante dont les grandes institutions républicaines, puissamment centralisées, font actuellement les frais. C'est dans ce contexte incertain de la modernité tardive marqué par le polythéisme des valeurs que le débat sur la laïcité a été réactivé en France et ce, depuis plusieurs années maintenant ${ }^{1}$. L'adversaire des républicains laïques a cependant radicalement changé: ce n'est (presque) plus l'antimodernisme structurel véhiculé par les clercs de l'Église catholique romaine, mais le zèle religieux et individuel de jeunes femmes musulmanes qui affichent de manière décomplexée leur appartenance religieuse dans l'espace public. Pour comprendre les revendications

1. V. Zuber, La laïcité en débat. Au-delà des idées reçues, Paris, Le Cavalier bleu, 2017. 
récentes en faveur d'une plus grande laïcité (également partagées par les protagonistes actuels du débat), il semble nécessaire de revenir sur l'histoire du concept et de son application en France. Seule une analyse historique peut nous permettre de comprendre la dialectique qui est à l'œuvre dans les débats récents: à la revendication d'une laïcité gage d'émancipation de l'individu qui est portée par le discours républicain s'oppose en effet la demande d'une laïcité-neutralité, respectueuse de la liberté de conscience, qui équivaudrait à une complète abstention de l'État en matière religieuse.

Jusqu'à la Révolution de 1789, les rois de France étaient les lieutenants de Dieu sur terre et concentraient dans leurs mains à la fois le pouvoir temporel et le pouvoir spirituel. Ils étaient sacrés dans la cathédrale de Reims (Charles X a été le dernier roi français sacré en 1825), et étaient les véritables chefs de l'Église catholique de France. Le catholicisme a été la seule religion officielle et autorisée de l'État français de 1685 (Édit de Fontainebleau qui abrogeait le pluralisme partiel légal instauré par l'Édit de Nantes de 1598) jusqu'à la veille de la Révolution (Édit de tolérance de 1787). À l'Église catholique romaine était dévolu l'encadrement de tous les aspects individuels et collectifs de la vie des Français: état-civil, enseignement, médecine... Le calendrier, les fêtes, les traditions populaires étaient, tous, d'inspiration catholique. Il n'empêche que le passé religieux français a souvent été tumultueux. L'histoire française a connu de graves schismes sectaires; de longues, violentes et fratricides guerres de religion entre catholiques majoritaires et protestants minoritaires ont eu lieu tout au long du XvI ${ }^{\mathrm{e}}$ siècle.

La position dominante de l'Église catholique a été fondamentalement remise en cause dès les premières semaines de la Révolution française. Pour les révolutionnaires, un principe majeur devait dominer la vie politique: l'exercice du pouvoir était le fruit d'un pacte raisonné entre les hommes (théorie du Contrat social de Jean-Jacques Rousseau) et la souveraineté émanait du et appartenait au peuple. «Le principe de toute souveraineté réside essentiellement dans la Nation»: par cet article 3 de la Déclaration des droits de l'homme et du citoyen de 1789, l'État est devenu totalement indépendant de l'Église. Les institutions n'étaient désormais plus justifiées par leur origine divine et incontestable, mais étaient créées et gouvernées par la volonté générale. La Déclaration reconnaissait aussi la liberté religieuse: «Nul ne doit être inquiété pour ses opinions mêmes religieuses, pourvu que leur manifestation ne trouble pas l'ordre public établi par la loi.» (art. 10)².

2. Déclaration des droits de l'homme et du citoyen, 1789: http://www.textes.justice.gouv.fr/ index.php? rubrique $=10086 \&$ ssrubrique $=10087 \&$ article $=10116$ [consulté le 11 févr. 2018] . 
Devant les résistances et les menaces, la Révolution a cependant très rapidement entamé une politique brutalement antireligieuse. Elle a nationalisé les biens du clergé, supprimé les ordres monastiques et les vœux religieux (car jugés contraires aux libertés contenues dans les droits de l'homme). Les membres du clergé, élus, devaient désormais prêter serment à la Constitution civile du clergé de $1790^{3}$. Certains prêtres, ne voulant pas désobéir au Pape qui avait formellement condamné cette constitution, refusèrent de prêter serment. L'Église catholique de France s'est alors divisée en deux parties inégales formées d'un côté par le clergé constitutionnel et de l'autre par le clergé réfractaire et hors la loi ${ }^{4}$. Des insurrections brutalement réprimées ont eu lieu en Vendée, région majoritairement réfractaire et royaliste. Dès 1792, une politique de laïcisation des institutions et une terrible répression antireligieuse se sont combinées qui ont entériné le divorce profond entre l'Église catholique et les idéaux révolutionnaires. Les actes de la vie civile (naissance, mariage, décès) ont alors été retirés à l'Église et les registres de l'état-civil transférés aux mairies. Le divorce a été autorisé, les obsèques pouvaient n'être que civiles, un calendrier républicain entièrement nouveau a été instauré (avec l'abandon des fêtes catholiques). Enfin, de nouvelles religions, tentatives d'expérimentation révolutionnaire de la religion civile conceptualisée par Jean-Jacques Rousseau ${ }^{5}$, sont brièvement apparues.

Au vu de l'échec patent du catholicisme constitutionnel et du culte de l'Être suprême à rassembler et surtout à pacifier les esprits, le décret du 3 ventôse an III (21 février 1795) ${ }^{6}$ a consacré la séparation de l'Église catholique et de l'État et réglementé l'exercice public des divers cultes: la République ne devait plus désormais subventionner aucun culte, mais le principe de liberté des cultes était rétabli. L'application en est restée cependant très partielle ${ }^{7}$. Lorsque Napoléon Bonaparte a pris le pouvoir, les rapports entre l'Église catholique romaine et le gouvernement étaient encore très conflictuels. L'Église catholique était toujours coupée en deux: une Église officielle boudée par les fidèles et une Église illégale, mais reconnue par le Pape qui encourageait la résistance aux mesures révolutionnaires.

3. Constitution civile du clergé du 12 juillet 1790 : https://fr.wikisource.org/wiki/Constitution_ civile_du_clerg\%C3\%A9_du_12_juillet_1790 [consulté le 11 févr. 2018].

4. T. TACKett, La Révolution, l'Église, la France, le serment de 1791, Paris, Cerf, 1986.

5. J.-J. Rousseau, «De la religion civile», Du Contrat social..., 1762, Livre IV, chapitre VIII.

6. Décret du 3 ventôse an III : http://www.eglise-etat.org/1795.html [consulté le 11 févr. 2018].

7. Cette histoire a été bien décrite dans l'ouvrage que Rita Hermon-Belot a consacré à l'abbé Grégoire: R. Hermon-Belot, L'abbé Grégoire, la politique et la véritée, Paris, Seuil, 2000. 
Afin d'obtenir la paix civile et religieuse, chaque partie a dû faire des concessions fixées par un contrat: c'est le Concordat de $1801^{8}$, qui mettait fin à l'expérience constitutionnelle et organisait les rapports entre l'Église catholique romaine et la République ${ }^{9}$. Le catholicisme n'était plus la religion de l'État, mais simplement celle de la «majorité des Français» et un pluralisme religieux partiel était légalisé par l'adjonction unilatérale des Articles organiques ${ }^{10}$ rédigés par le conseiller d'État de Napoléon Bonaparte, Jean-Étienne-Marie Portalis. C'est ce qu'on a appelé le régime des «cultes reconnus ». Quatre cultes étaient concernés (catholicisme, calvinisme, luthéranisme, et un peu plus tard, le judaïsme), les autres étant simplement tolérés. Le pouvoir civil nommait les évêques, versait un salaire aux évêques et aux curés (et par la suite aux pasteurs et aux rabbins). En retour, les membres des différents clergés devaient prêter serment et accepter une police des cultes particulièrement tatillonne. Avec le Concordat, on a assisté à un certain recul de la laïcité dans ses formes les plus radicales, au bénéfice de la reconnaissance légale d'une certaine pluralité religieuse ${ }^{11}$. Napoléon reconnaissait l'Église catholique romaine, mais ne s'y soumettait pas. Son couronnement a eu lieu en présence du pape, mais sans qu'il y ait eu de sacre. Le mariage civil et le divorce ont été maintenus, et un Code civil sans aucune mention religieuse a été institué (1804). En revanche, le calendrier républicain a été définitivement supprimé (1806).

Ce régime des cultes reconnus a connu une longévité exceptionnelle (un peu plus d'un siècle de fonctionnement) et a survécu à tous les virages politiques du XIX ${ }^{e}$ siècle. Les rapports entre l'État et les Églises ont cependant varié selon l'attitude plus ou moins anticléricale adoptée par les différents régimes vis-à-vis du catholicisme romain et de son clergé ${ }^{12}$. Sous la Restauration (1814-1830), le parti clérical légitimiste a retrouvé son influence et des mesures hautement symboliques ont été prises. Le catholicisme a été à nouveau qualifié dans la Charte de «religion de l'État ${ }^{13}$, même si la pluralité

8. Loi du 18 germinal an X (8 avril 1802) relative à l'organisation des cultes.

9. J.-O. Boudon (éd.), Le Concordat et le retour à la paix religieuse, Actes du colloque organisé par l'Institut Napoléon et la Bibliothèque Marmottan, 13 oct. 2001, Paris, SPM, 2008.

10. Articles organiques de la Convention du 26 Messidor an IX: https://fr.wikisource.org/ wiki/Concordat_de_1801 et http://www.droitcanon.com/Articles_organ.html [consultés le 11 févr. 2018].

11. J.-M. Leniaud, L'Administration des cultes pendant la période concordataire, Paris, NEL, 1988.

12. B. BASDEVAnt-Gaudemet, Le jeu concordataire dans la France du XIXe siècle. Le clergé devant le Conseil d'État, Paris, PUF, 1988.

13. Charte constitutionnelle du 4 juin 1814: http://www.conseil-constitutionnel.fr/ conseil-constitutionnel/francais/la-constitution/les-constitutions-de-la-france/charteconstitutionnelle-du-4-juin-1814.5102.html [consulté le 11 févr. 2018]. 
des cultes était maintenue. Le divorce est interdit (8 mai 1816) et une loi punissant le sacrilège a été votée par la Chambre des pairs (20 avril 1825). Sous la Monarchie de Juillet (1830-1848), on s'est empressé de supprimer la plupart des inflexions cléricales prises sous le régime précédent. Selon la nouvelle Charte constitutionnelle, la religion catholique redevenait la religion «professée par la majorité des Français ». L'égalité juridique entre les cultes reconnus était rétablie et la loi sur le sacrilège supprimée. Avec la Révolution de février 1848, une fraternité nouvelle a semblé rapprocher les anciens ennemis. Le clergé catholique a béni les arbres de la liberté et les insurgés se sont spontanément agenouillés devant le Saint-Sacrement. La Constitution a rétabli la liberté de culte et l'égalité des religions a été solennellement proclamée. Cette réconciliation n'a cependant pas résisté aux Journées de juin et la guerre des deux France a repris de plus belle avec le vote de la Loi Falloux sur l'enseignement, qui renforçait le contrôle des curés et des notables sur l'école (15 mars 1850). Sous le Second Empire (1852-1870), Napoléon III s'est d'abord appuyé sur le parti clérical et lui a donné de sérieuses garanties par sa politique étrangère en faveur du maintien des États pontificaux. Le conflit des deux France s'est encore radicalisé. Il semblait à beaucoup qu'il n'y avait plus moyen de réconcilier un catholicisme romain de plus en plus intransigeant, soutien du régime, et un parti républicain d'opposition qui est peu à peu passé de l'anticléricalisme traditionnel à l'antireligion déclarée ${ }^{14}$. Même si la politique cléricale s'est beaucoup ralentie dans les dernières années de l'Empire, l'exaspération était si forte chez certains qu'elle explique en partie la fureur anticléricale qui s'est manifestée lors des événements tragiques de la Commune (l'archevêque de Paris a ainsi été exécuté sommairement par les insurgés en 1871). Après la défaite militaire de 1871 et la chute du Second Empire, la République (amputée de l'Alsace-Moselle au profit de l'Allemagne) a certes été rétablie, mais restait fragile et sujette à tout changement de majorité qui aurait rendu possible le rétablissement de la monarchie. Par un souci d'apaisement du conflit, la $\mathrm{III}^{\mathrm{e}}$ République (1870-1877) n'a pas opéré, à ses débuts, de changements notables en ce qui concerne la laïcité de l'État.

Aux élections de 1879, les Républicains l'ont largement emporté sur leurs adversaires monarchistes. De nombreuses lois fondamentales qui allaient dans le sens d'une plus grande laïcisation de l'État ont alors été adoptées en moins d'une dizaine d'années: suppression de l'obligation du repos dominical (12 juin 1880), interdiction des congrégations et expulsion de la Compagnie

14. V. la typologie des anticléricalismes français in R. RÉMOND, L'anticléricalisme en France de 1815 à nos jours, Bruxelles, Complexe, 1985. 
de Jésus (29 mars 1880), sécularisation des cimetières (14 novembre 1881), laïcisation de l'école primaire (28 mars 1882), suppression des prières publiques à la Chambre des députés (14 août 1884) et rétablissement du divorce (27 juillet 1884), suppression des facultés théologiques d'État et laïcisation des hôpitaux (21 mars 1885), laïcisation du personnel d'enseignement dans les établissements laïques (30 octobre 1886), enlèvement des emblèmes religieux dans les tribunaux et liberté des funérailles (15 novembre 1887), astreinte des séminaristes et des clercs au service militaire (15 juillet 1889).

Cette politique volontariste était certes d'inspiration anticléricale, mais pas foncièrement antireligieuse. Jules Ferry, alors président du Conseil, athée convaincu, était cependant très soucieux de ne pas heurter trop profondément les convictions de la France croyante. Mais la rapidité de l'exécution du programme de laïcisation a réactivé les rivalités entre les tenants des «deux France». Les conflits sont redevenus très violents, en dépit de l'apaisement prôné par le Pape Léon XIII avec son appel aux catholiques français afin qu'ils se «rallient» à la République (Encyclique Inter sollicitudines du 20 février 1892). L'affaire Dreyfus qui a alors surgi en divisant gravement les Français a encore radicalisé les oppositions. Au tournant du siècle, certains ont parfois eu l'impression d'être à la veille d'une véritable guerre civile et religieuse. Une série de conflits, exacerbés par les lois très sévères prises à l'encontre des congrégations religieuses ( $1^{\text {er }}$ juillet 1901 et 7 juillet 1904) ${ }^{15}$, ont finalement conduit à la rupture des relations diplomatiques avec le Saint-Siège (1904). C'était la fin du Concordat, la séparation des Églises et de l'État devenait possible. Les principaux artisans de la loi qui a été finalement votée le 9 décembre 1905 après de nombreux débats ont cherché à «assurer la pacification des esprits » et à offrir aux Églises «la possibilité de vivre à l'abri de ce régime ${ }^{16} \gg$. Les deux articles établissant les principes majeurs de la loi sont l'article 1: «La République assure la liberté de conscience. Elle garantit le libre exercice des cultes sous les seules restrictions édictées ci-après dans l'intérêt de l'ordre public» et l'article 2: "La République ne reconnaît, ne salarie ni ne subventionne aucun culte ${ }^{17}$.

15. J. Lalouette, J.-P. Machelon (éd.), Les Congrégations hors la loi? Autour de la loi du

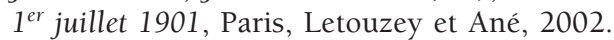

16. M. LaRkin, L'Église et l'État en France. 1905: la crise de la Séparation, Toulouse, Privat, 2004, trad. de Church and State after the Dreyfus Affair. The Separation Issue in France, London, Macmillan, 1974.

17. Loi du 9 décembre 1905 concernant la séparation des Églises et de l'État: https:// www.legifrance.gouv.fr/affichTexte.do?cidTexte=LEGITEXT000006070169 [consulté le 11 févr. 2018]. 
Cette loi a donc signé la fin du système napoléonien des cultes reconnus, en mettant toutes les Églises à égalité devant l'État. L'État a mis à la disposition gratuite des Églises les lieux de culte et, avec les départements et les communes, a continué à assurer l'entretien des bâtiments sur fonds publics. Il ne devait plus y avoir de signes ou d'emblèmes religieux sur les monuments autres que religieux ou dans les lieux publics. Ce sont les communes qui devaient gérer la sonnerie des cloches. Les Églises devaient s'organiser sous la forme d'associations cultuelles et subvenir financièrement à leurs besoins. Elles n'avaient plus de service public à assurer. Si les protestants ${ }^{18}$ et les juifs acceptèrent assez facilement la loi de 1905, la majorité des catholiques la refusèrent. De sérieuses difficultés d'application ont surgi lors des inventaires des biens des églises prévus par la loi. Dans certaines régions, on a même observé des résistances à la police et des affrontements. Le pape Pie X a finalement condamné le principe de séparation, et ordonné à ses fidèles de ne pas créer les associations cultuelles prévues par la loi (encycliques Vehementer Nos et Gravissimo Officii Munere du 11 février et 10 août 1906). Un an après la promulgation de la loi, les lieux de cultes catholiques devaient revenir à l'État et aux communes. Était-ce la grande expropriation annoncée par les détracteurs de la loi? Non. Dans un souci d'apaisement, le gouvernement a fait passer trois lois complémentaires qui laissaient gratuitement et sans arrangements contractuels l'usage des biens ecclésiastiques au clergé catholique ( 2 janvier, 28 mars 1907 et 13 avril 1908) ${ }^{19}$.

Pendant la Première Guerre mondiale, les conflits d'avant-guerre se sont estompés face aux nécessités de l'Union sacrée. Prêtres et congréganistes se sont engagés en masse pour la défense de la Patrie. En 1921 les relations diplomatiques de l'État français avec le Saint-Siège ont été rétablies et un compromis a été finalement trouvé (accords Briand-Ceretti du 11 janvier 1924): le pape Pie XI autorisait la formation d'associations cultuelles aménagées dans le respect de la hiérarchie catholique: les associations diocésaines (encyclique Maximam Gravissimamque du 18 janvier 1824) ${ }^{20}$. En 1945, l'Église catholique accepta officiellement la laïcité désormais constitutionnalisée.

18. P. Harizmendy (éd.), «Les protestants et la séparation des Églises et de l'État», Bulletin de la Société de l'histoire du protestantisme français, vol. 151, oct.-nov.-déc. 2005.

19. Loi du 2 janvier 1907 concernant l'exercice public du culte, loi du 28 mars 1907 relative aux réunions publiques et loi du 13 avril 1908 modifiant les articles 6, 7, 9, 10, et 14 de la loi de 1905: accessibles sur http://www.legifrance.gouv.fr/ [consultés le 11 févr. 2018].

20. É. Poulat, Les Diocésaines, République française, Église catholique: Loi de 1905 et associations cultuelles, le dossier d'un litige et de sa solution (1903-2003), Paris, La Documentation française, 2007. 
Depuis sa signature, la loi de 1905 a subi quelques modifications, mais ses grands principes ont toujours été maintenus. La laïcité a été inscrite pour la première fois dans la Constitution du 27 octobre 1946, dans l'article $1^{\mathrm{er}}$ : «La France est une République indivisible, laïque, démocratique et sociale » et on peut aussi lire dans son préambule que: «L'organisation de l'enseignement public, gratuit et laique à tous les degrés est un devoir de l'État». La République se déclarait clairement laïque. La Constitution du 4 octobre 1958 a repris le préambule de 1946, et a par ailleurs réaffirmé que la République « respecte toutes les croyances».

\section{LA LAÏCITÉ FRANÇAISE, UN MODÈLE PARMI D'AUTRES}

La laïcité est un principe partagé: certains commentateurs arguent de l'exceptionnalité française en matière de laïcité en rendant compte de la difficulté qu'il y aurait à traduire le terme de laïcité dans d'autres langues. Le terme de laïcité trouve pourtant de nombreuses correspondances dans la plupart des langues, latines ou non (laicismo pour l'espagnol, mais aussi Laïzismus pour l'allemand ou laiklik pour le turc). D'autre part, l'idée contenue par le principe de laïcité, rendue par le néologisme de sécularité (ou État séculier) se traduit fort bien dans la plupart des langues (secular state pour l'anglais, secularizare pour le roumain, Säkularismus pour l'allemand). Il paraît difficile d'admettre que l'absence d'un terme rigoureusement équivalent au terme français dans les autres langues conclurait nécessairement à une méconnaissance de ce principe en dehors de frontières strictement nationales. Les autres langues font en tout cas bien la différence entre deux acceptions du terme de laïcité, dangereusement confondues en français: la laïcité comme principe juridico-politique (rendu par le terme de secularity en anglais et de laicità en italien), et la laïcité comme idée philosophique athée (secularism en anglais, secularismo en italien).

Le modèle français de séparation des Églises et de l'État, issu de la loi de 1905, n'est qu'un modèle de laïcité parmi d'autres ${ }^{21}$. Par ailleurs, loin d'être une invention française, ce modèle séparatiste a été instauré légalement aux États-Unis d'Amérique un siècle et demi avant la France. C'est en effet le premier amendement à la Constitution fédérale adopté en 1791 qui, en plus de la liberté d'expression, de la presse et d'association garantie à tous, énonce que «le Congrès n'adoptera aucune loi relative à l'établissement

21. V. Zuber, La laïcité en France et dans le monde, Paris, La Documentation française, 2017. 
d'une religion, ou à l'interdiction de son libre exercice». En 1802, Thomas Jefferson écrivait que la Constitution américaine avait, selon lui, créé «un mur de séparation entre l'Église et l'État». Celui-ci ne s'appliquait jusqu'alors qu'à l'État fédéral, les États fédérés restant libres du choix de leurs relations stato-religieuses. Mais en 1868, le quatorzième amendement à la Constitution a finalement consacré la séparation des Églises et de l'État dans tous les États américains sans exception. Il est par ailleurs strictement interdit à l'État de financer quelques manifestations ou organisations religieuses que ce soit.

De la même façon, le Mexique a devancé la France (et lui a même servi de modèle lors de l'abandon du système concordataire napoléonien au tout début du $\mathrm{xx}^{\mathrm{e}}$ siècle). Les "lois de la Réforme» conduites par Benito Juarez (1859-1863) ont organisé la séparation de l'Église et de l'État cinquante ans avant la France. Le gouvernement mexicain a ainsi mis fin à la république catholique précédente en supprimant le financement des cultes et en instaurant la liberté religieuse pour tous. Il a également nationalisé les biens de l'Église catholique et institué des registres d'état-civil indépendants de ceux tenus jusque-là par les clercs. En 2012, le principe de laïcité a été définitivement inscrit dans la Constitution mexicaine faisant de ce pays une «République représentative, démocratique, laĩque et fédérale».

D'autres modèles de laïcité existent dans le monde, incluant les formes - historiquement héritées - des pays à religion d'État (Angleterre, Danemark, Grèce...). Même dans ces pays, dont le chef est généralement aussi le responsable de l'Église nationale, la liberté religieuse, garantissant la liberté de conscience et le libre exercice du culte, est accordée à tous les individus et toutes les communautés religieuses présentes sur le territoire. L'égale citoyenneté et la non-discrimination pour des motifs religieux de leurs membres est la règle absolue. Cependant, la majorité des États laiques européens et mondiaux pratiquent plutôt une forme de reconnaissance légale des différentes religions, allant jusqu'à leur éventuel subventionnement par l'État selon des critères variés se rapportant à l'histoire du pays, l'ancienneté de leur ancrage territorial, à leur représentativité d'un point de vue quantitatif et à leur adhésion avec le pluralisme de la société et le fonctionnement démocratique de l'État. C'est le cas de l'Allemagne qui par sa Loi fondamentale reconnaît aux deux grandes confessions chrétiennes des prérogatives étatiques en matière de santé et d'éducation. C'est aussi le cas de l'Italie, de l'Espagne et des pays nouvellement intégrés à l'est du continent, qui ont mis en place des systèmes de conventions avec les différentes religions existant sur leur territoire. C'est enfin le cas de la France, qui entretient des relations 
contractuelles avec certaines religions reconnues en Alsace-Moselle, ou bien dans certains départements d'outre-mer.

La laïcité d'un État se mesure en effet moins à son organisation socio-religieuse ou à sa forme politique qu'au respect de plusieurs critères constitutifs de l'État de droit. Dans ce cadre, la neutralité de l'État interdit à ce dernier d'interférer en quoi que ce soit dans l'élaboration ou le contenu des doctrines religieuses, mais aussi de s'ingérer dans l'organisation interne des Églises. Il doit aussi être, nous l'avons dit, le garant du respect de la liberté de religion et de conviction de tous et s'assurer que l'égalité civile de tous les individus, indépendamment de leur éventuelle appartenance religieuse, est réelle. Le principe de laiicité de l'État séculier oblige ce dernier à respecter scrupuleusement l'indépendance des instances et décisions politiques vis-à-vis des normes religieuses. Inversement, les organisations religieuses doivent s'abstenir de contester ce qui relève du domaine de l'État et sont dans l'obligation de reconnaître la supériorité des lois civiles sur les lois religieuses.

La laïcité est une notion extrêmement polymorphe, à la fois dans le temps et dans l'espace, et c'est ce qui explique en partie les nombreux et incessants débats qu'elle provoque. La Laïcité (avec un grand L) n'existe donc pas, il n'y a que des formes diverses et contextualisées de laïcité, issues de processus historiques de laïcisation, tous distincts. La laïcité n'est jamais un acquis définitif, elle ne cesse de se transformer (soit en se renforçant, soit en s'étiolant suivant la coloration des décisions prises par les gouvernements politiques). En ce sens, les chercheurs décrivent plus volontiers des degrés différents dans le processus de laïcisation de tel ou tel pays que de laïcité en tant que système achevé. Ce qui permet de qualifier et de mesurer le degré de laïcité dans un État, c'est bien plus l'évaluation de son degré d'inscription dans un processus de laïcisation juridique qui est toujours un processus en cours. 